\title{
the ATLAS Experiment
}

ATLAS Silicon Microstrip Tracker Operation and Performance

Anna Mayne

University of Sheffield

ATLAS Collaboration 


\section{The ATLAS Detector}

- Inner Detector

Composite tracking system: Pixel detector, SemiConductor Tracker (SCT) and Transition Radiation Tracker (TRT)

Full coverage in $\phi$ and acceptance in pseudorapidity $|\eta|<2.5$

Solenoid provides a $2 \mathrm{~T}$ magnetic field

- Calorimeters

EM calorimeter: Liquid Ar Hadronic calorimeter: Liquid Ar endcap Tile calorimeter (Fe scintillator) barrel

- Muon Spectrometers

- Toroid provides a $4 \mathrm{~T}$ field for muon tracking

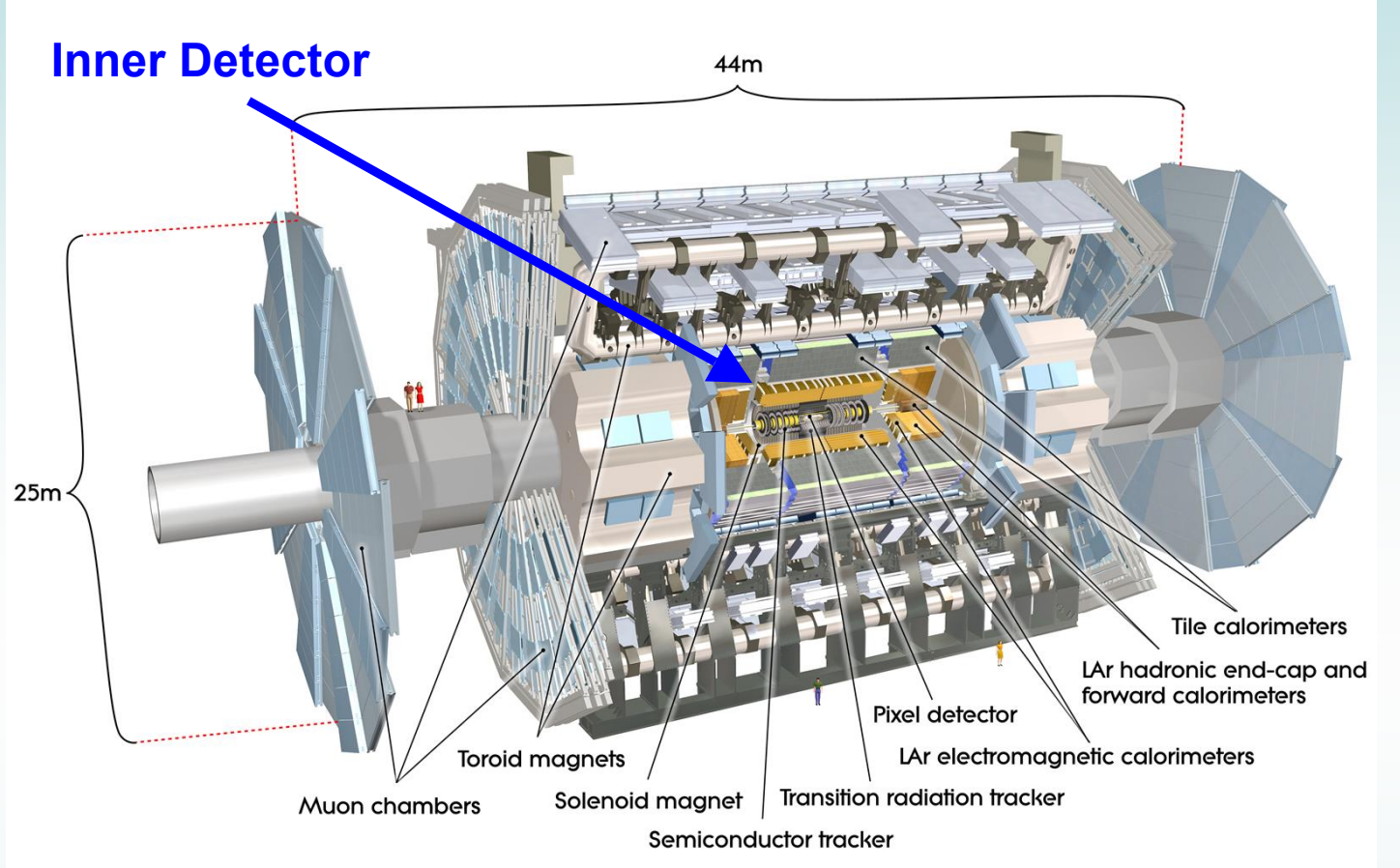

- Triggers

3 level trigger system:

Level 1 (hardware) reduces the accept rate to $75 \mathrm{kHz}$.

$2.5 \mu$ s latency (decision time)

Level 2 and Event Filter (software) reduces the acceptance rate further to up to $200 \mathrm{~Hz}$ (latency of $10 \mathrm{~ms}$ and $2 \mathrm{~s}$ respectively)

Anna Mayne - IPRD2010 


\section{The Inner Detector}

Designed to accurately reconstruct vertices and charged particle tracks

- Pixel

3 cylindrical pixel-silicon layers and $2 \times 3$ endcap disks 1744 modules, each containing 47,232 pixels

Pixels mostly $50 \mu \mathrm{m} \times 400 \mu \mathrm{m}$

- SCT

Sandwiched between Pixel and TRT

- TRT

298,304 drift tubes (straws)

3 cylindrical layers with polypropylene fibres in the barrel between the straws

80 wheel-like endcap disks with polypropylene foils between the straws

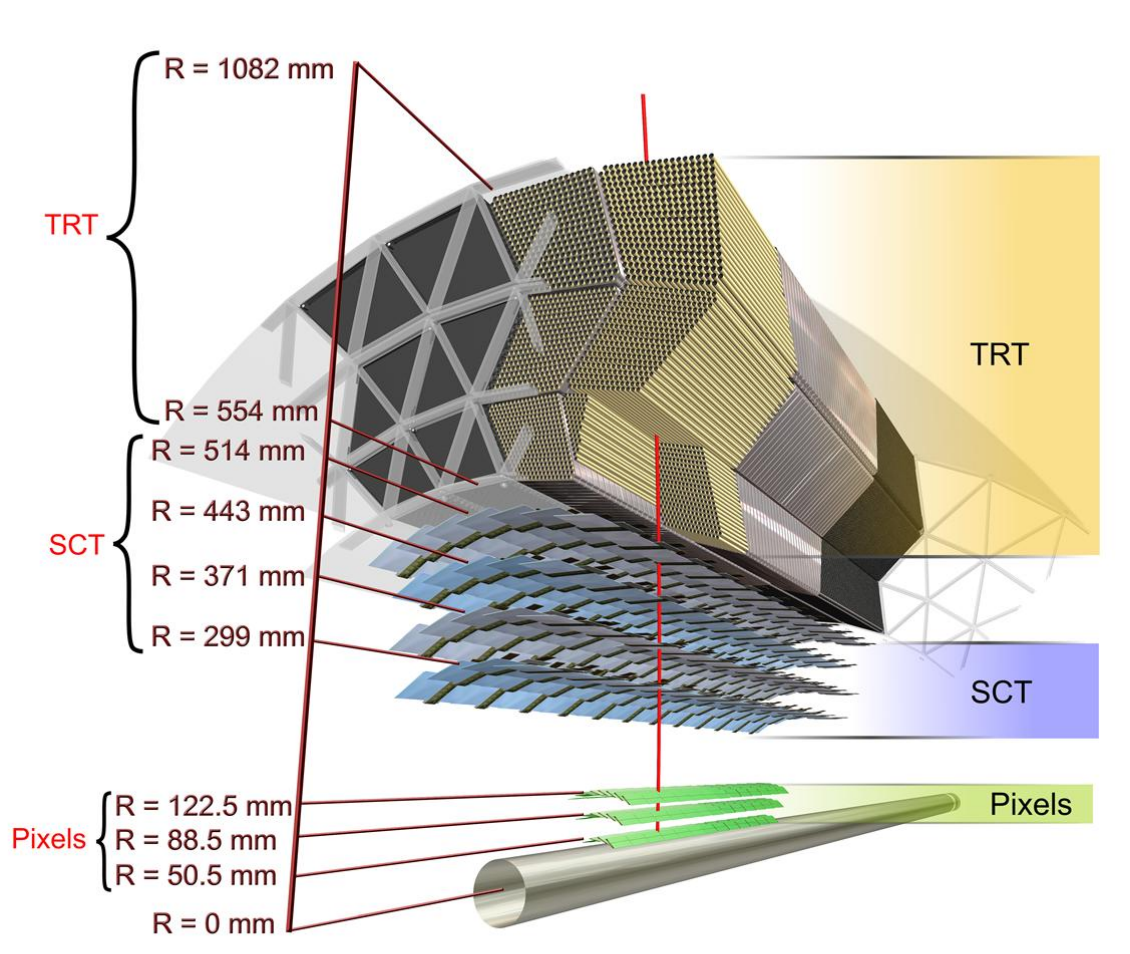

\section{Design Resolution}

Transverse momentum resolution:

$$
\sigma\left(p_{\mathrm{T}}\right) / \mathrm{p}_{\mathrm{T}}=0.05 \% \mathrm{p}_{\mathrm{T}} \mathrm{GeV} \oplus_{1} \%
$$

Transverse impact parameter resolution:

$$
\sigma\left(d_{0}\right)=10 \mu \mathrm{m} \oplus 140 \mu \mathrm{m} / \mathrm{p}_{\mathrm{T}}(\mathrm{GeV} / \mathrm{c})
$$




\section{The SCT}

- 4 concentric barrel layers and 2 x 9 endcap disks

- Barrel: $|z|<805$ mm, $299<R<514$ mm

- Endcap: $810<|z|<2797 \mathrm{~mm}, 251<\mathrm{R}<610 \mathrm{~mm}$

- 4088 silicon strip detector modules

- 2112 Barrel Modules, 1976 Endcap Modules

- Cooled with $\mathrm{C}_{3} \mathrm{~F}_{8}$ fluid delivered to detector with temp $<0{ }^{\circ} \mathrm{C}$

- $2 \times 768$ strips per module, $\sim 6.3$ million strips total

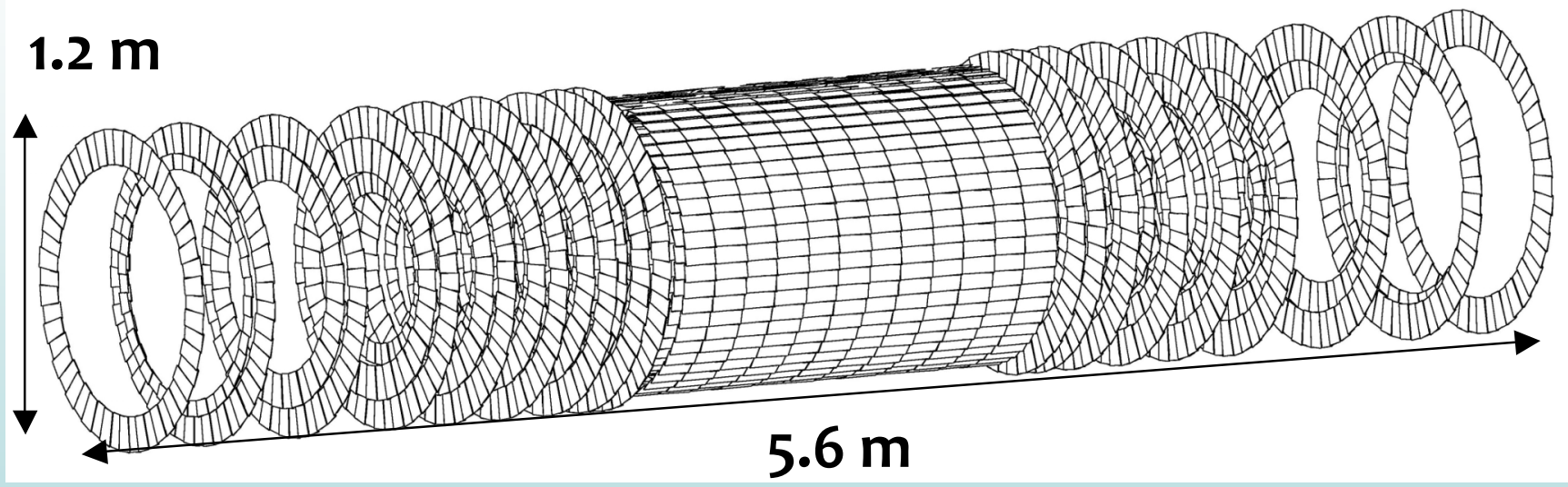




\section{SCT Modules}

- $p$-in-n single sided silicon-strip sensors $(\sim 6 \mathrm{~cm}$ in length)

- Barrel module strip length $\sim 12 \mathrm{~cm}$, pitch $80 \mu \mathrm{m}$

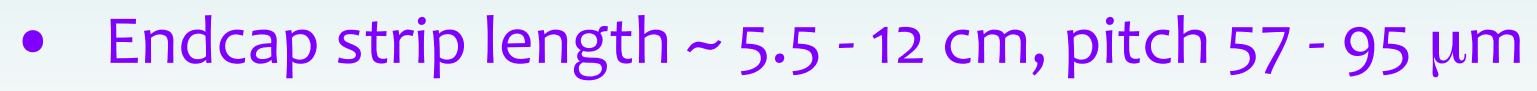

- Sensors glued back-to-back at a stereo angle of $40 \mathrm{mrad}$, allowing for a 2-dimensional position measurement

- Sensors separated by a thermal pyrolitic graphite (TPG) base-board/spine

- The base-board/spine provides the thermal and mechanical structure

- Intrinsic accuracy per module: $17 \mu \mathrm{m}(\mathrm{R}-\phi)$ and $580 \mu \mathrm{m}(\mathrm{z})$ for barrel, $580 \mu \mathrm{m}(\mathrm{R})$ for endcap
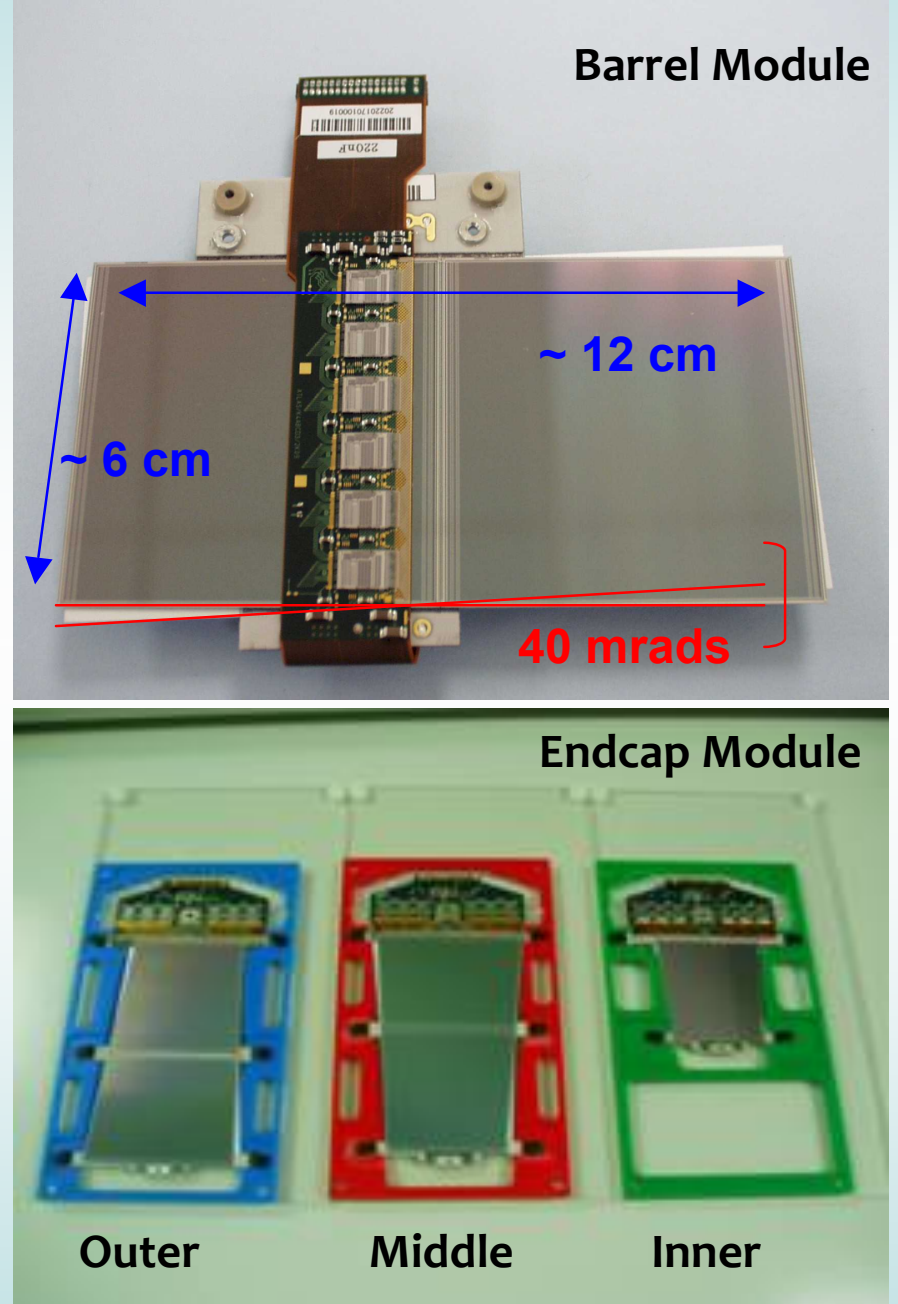


\section{SCT Module Readout}

- Strips are read out by radiation-hard frontend readout chips ( $\left.A B C D_{3} \mathrm{~T}\right)$

- 6 readout chips on each side of module, each responsible 128 channels

- Readout is binary

- Hit or no hit (1 or 0) provided and stored for each of the 128 channels of the chip
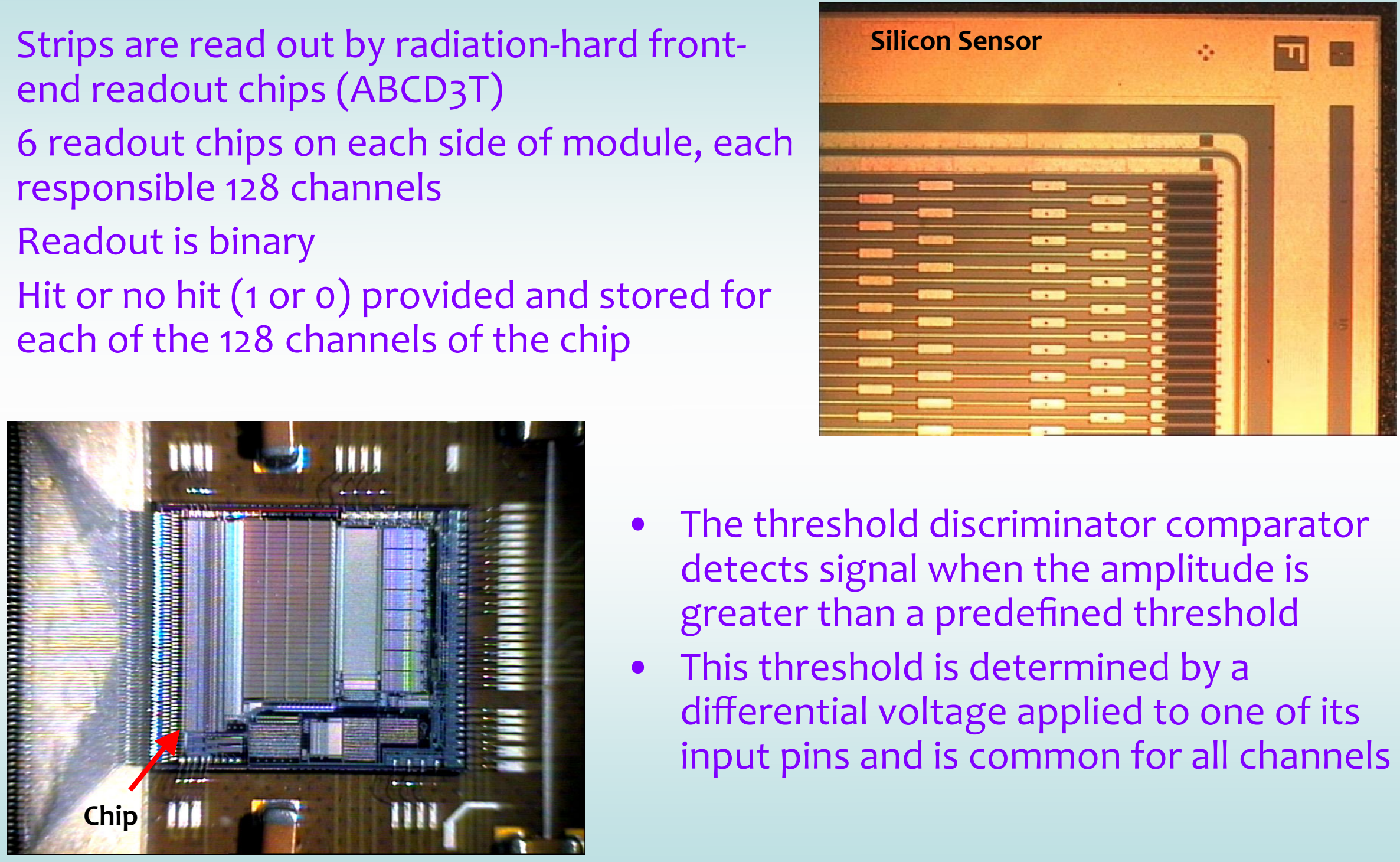

- The threshold discriminator comparator detects signal when the amplitude is greater than a predefined threshold

- This threshold is determined by a differential voltage applied to one of its input pins and is common for all channels 


\section{Design Requirements}

- Inner Detector tracking efficiency: Reconstruct isolated leptons with $\mathrm{p}_{\mathrm{T}}>5 \mathrm{GeV}$ with $95 \%$ efficiency out to $|\eta|<2.5$

- SCT strip efficiency $>99 \%$

- Noise Occupancy $<5 \times 10^{-4}$ per bunch crossing Required at a noise threshold of $1 \mathrm{fC}$ (the nominal operational value of modules)

- Maximum of $1 \%$ of strips allowed to be defective 


\section{Calibration}

- S curve (nHits vs threshold) produced by varying threshold whilst injecting a fixed signal

- Threshold at which occupancy is $50 \%=V_{t 50}$

- Distribution shows r.m.s spread of the threshold $V_{t 50}$ for each chip in SCT barrel.

- Trim range corresponds to TrimDAC setting which allows correction of individual channel thresholds in order to maintain uniformity of response

- Uniformity of response 4\%

- S curve is described by a complementary error function

- Derivative of error function is the noise distribution

- $\sigma$ of this distribution = noise after amplification

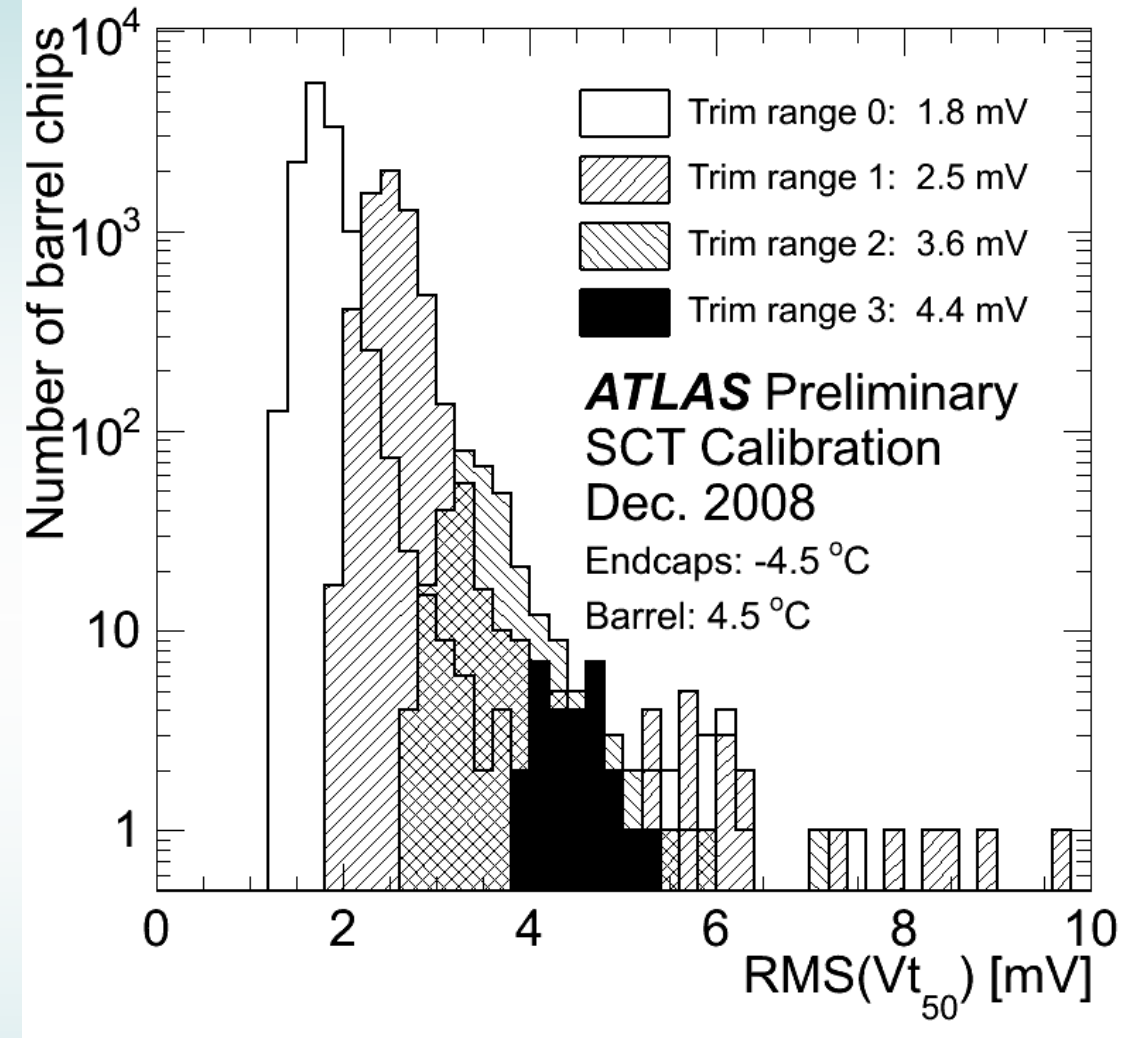

Calibration carried out during stand alone calibration runs in $\mathbf{2 0 0 8}$ 


\section{Calibration}

Noise

- Input noise values for each chip as obtained in response curve tests

- SCT noise 1500 e, well below typical threshold set at $1 \mathrm{fC}$ $(1 \mathrm{fC} \sim 6,240 \mathrm{e})$

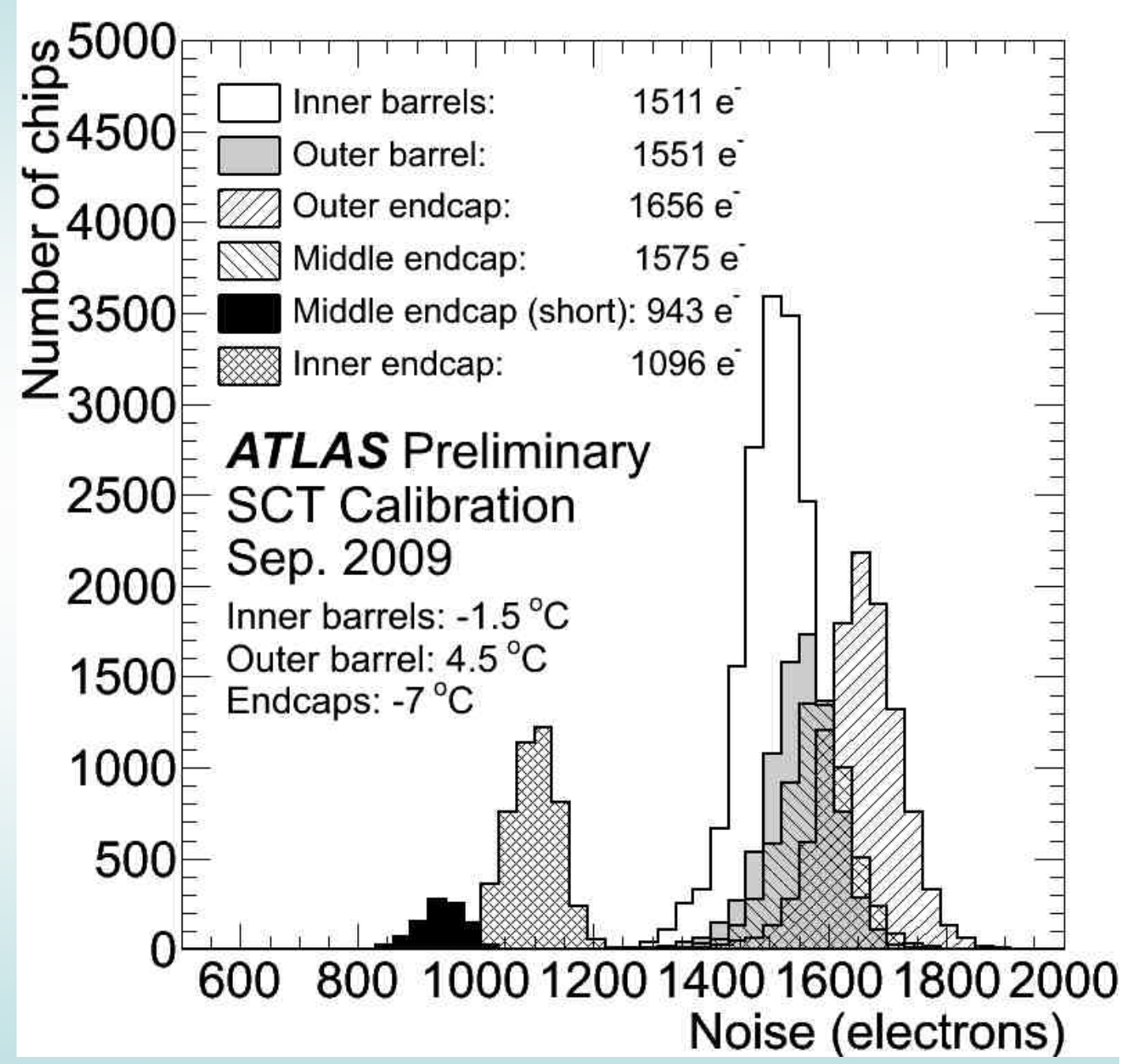




\section{Calibration}

- Noise occupancy specification:

$$
<5 \times 10^{-4}
$$

- Can be measured from noise using charge injection or directly counting hits using a random trigger

- Both methods show good agreement
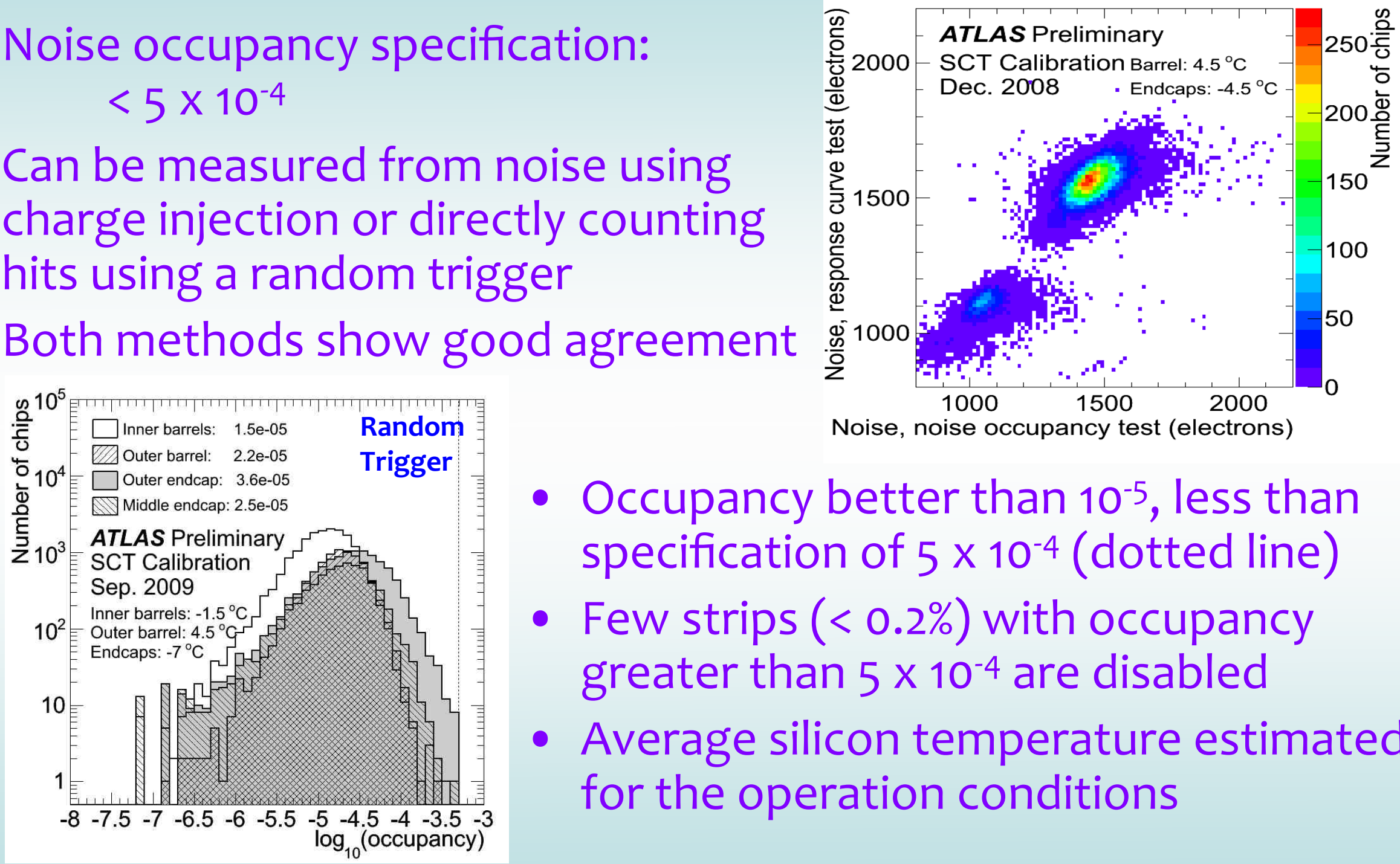

- Occupancy better than $10^{-5}$, less than specification of $5 \times 10^{-4}$ (dotted line)

- Few strips (<0.2\%) with occupancy greater than $5 \times 10^{-4}$ are disabled

- Average silicon temperature estimated for the operation conditions 


\section{Path to Collisions}

\section{8}

- Single-beam LHC running

Useful for commissioning SCT (timing, efficiency measurements, Noise Occupancy etc)

- September setback

- Combined ATLAS cosmic-ray run

- Standalone ID cosmic-ray run

Cosmics extremely useful for ID alignment and detector timing and for testing the triggers, tracking and data acquisition system

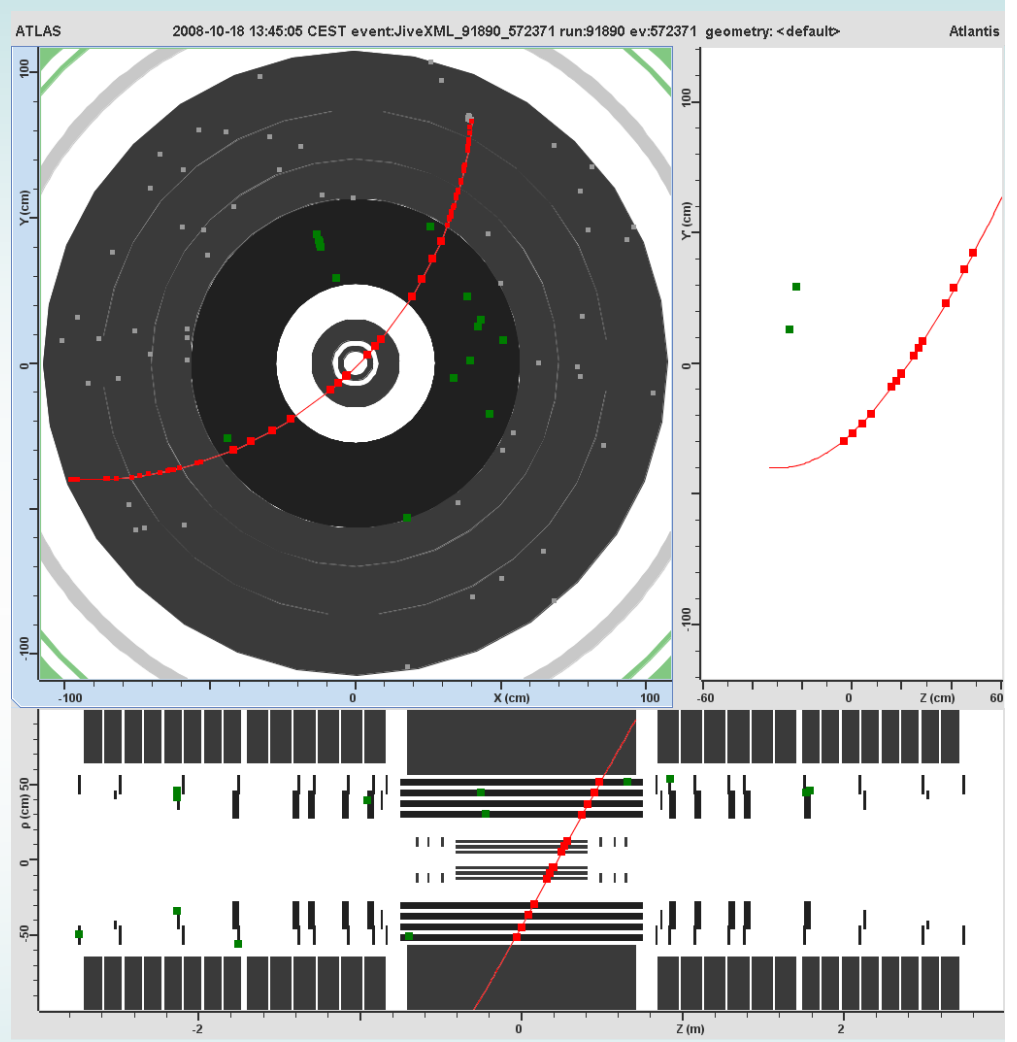

Inner Detector event display of a cosmic event 


\section{Path to Collisions}

2009:

- More cosmic runs
(November $23^{\text {rd }}$ ):

- First collisions at $900 \mathrm{GeV}$ !

\section{(December $8^{\text {th }}$ ):}

- $2.36 \mathrm{TeV}$ collisions (world record)
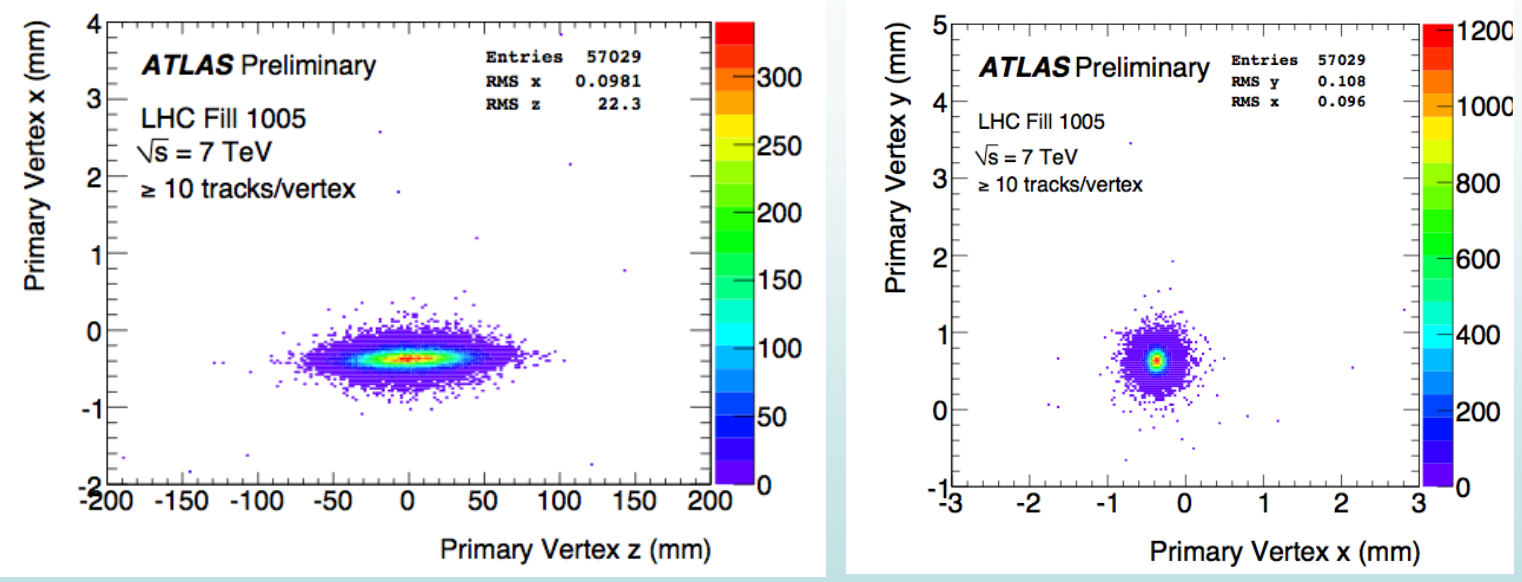

the ATLAS Experiment Cand

Anna Mayne - IPRD2010

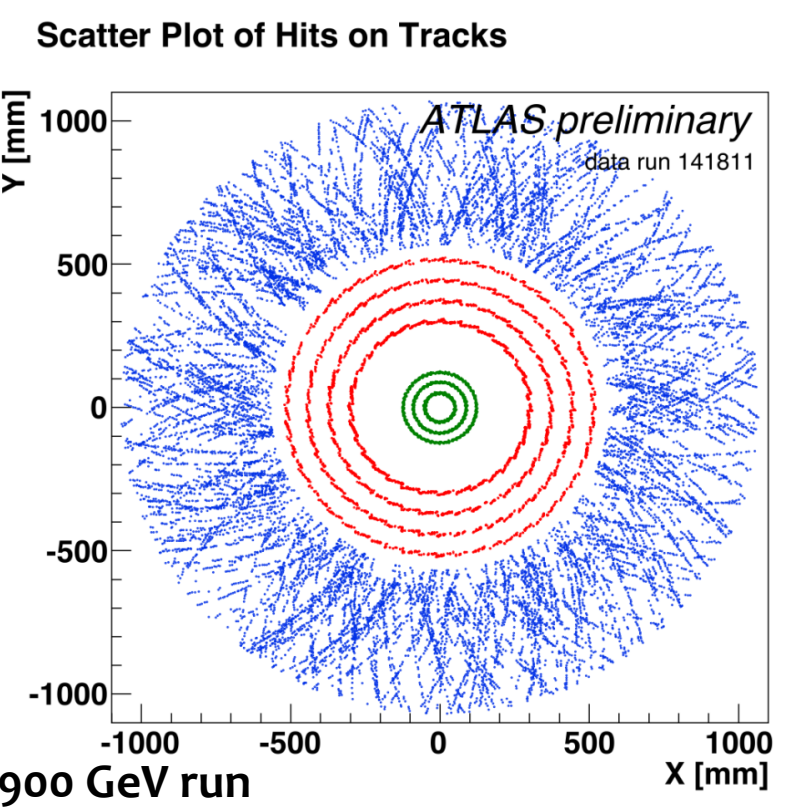

\section{0}

- 932 million $7 \mathrm{TeV}$ collisions

- ATLAS recorded lumi: $16.02 \mathrm{nb}^{-1}$ as of $08 / 06 / 2010$ 


\section{Operating Conditions}

- Detector Control System (DCS) controls and monitors the supply of powering, cooling and other control signals, ensuring a safe functionality

- Most of detector operational during cosmic runs

- Configuration stable since 2009

- Currently 99.3\% of all SCT modules are operational

\begin{tabular}{l|ccccc}
\multicolumn{6}{c}{ ATLAS SCT Configuration May 2010 } \\
\hline $\begin{array}{l}\text { Disabled Readout } \\
\text { Components }\end{array}$ & Endcap A & Barrel & Endcap C & SCT & $\begin{array}{c}\text { Fraction } \\
(\%)\end{array}$ \\
\hline Disabled Modules & 5 & 10 & 15 & 30 & 0.73 \\
Disabled Chips & 5 & 24 & 4 & 33 & 0.07 \\
Masked Strips & 3,364 & 3,681 & 3,628 & 10,673 & 0.17 \\
\hline Total Disabled & & & & & 0.97 \\
Detector Region & & & & & \\
\hline
\end{tabular}

\section{ATLAS SCT Disabled Modules May 2010}

\begin{tabular}{l|ccccc}
\hline & Endcap A & Barrel & Endcap C & SCT & $\begin{array}{c}\text { Fraction } \\
(\%)\end{array}$ \\
\hline Total & 5 & 10 & 15 & 30 & 0.73 \\
Fraction (\%) & 0.5 & 0.2 & 1.5 & 0.7 & \\
\hline Cooling & 0 & 0 & 13 & 13 & 0.32 \\
LV & 0 & 6 & 1 & 7 & 0.17 \\
HV & 4 & 1 & 1 & 6 & 0.15 \\
Readout & 1 & 3 & 0 & 4 & 0.10 \\
\hline
\end{tabular}

- Operational strips better than specification 


\section{Performance}

Timing

- Readout of the SCT is synchronized with the bunch-crossing time

- Ensures that the signal is sampled at the peak of the charge response curve

- SCT reads out three consecutive 25 ns time bins (o, 1 and 2). 25 ns corresponds to LHC bunch crossing spacing

- Readout is centered on time bin 1 (same bunch-crossing as level-1 accept)

- Hits corresponding to tracks from a collision should have nothing in the first bin and should be over the threshold in the second bin. No requirement on third bin

- Average of time bin value (0,1 or 2$)$ taken over all events for all layers 


\section{Performance}

\section{Timing}

- Commissioned with a threshold of $1 \mathrm{fC}$

- The mean time bin should ideally be between 1 and 2

- For this plot individual module timing offsets were adjusted for optical fibre lengths

- Timing is very good/uniform without final adjustments from fine timing scan

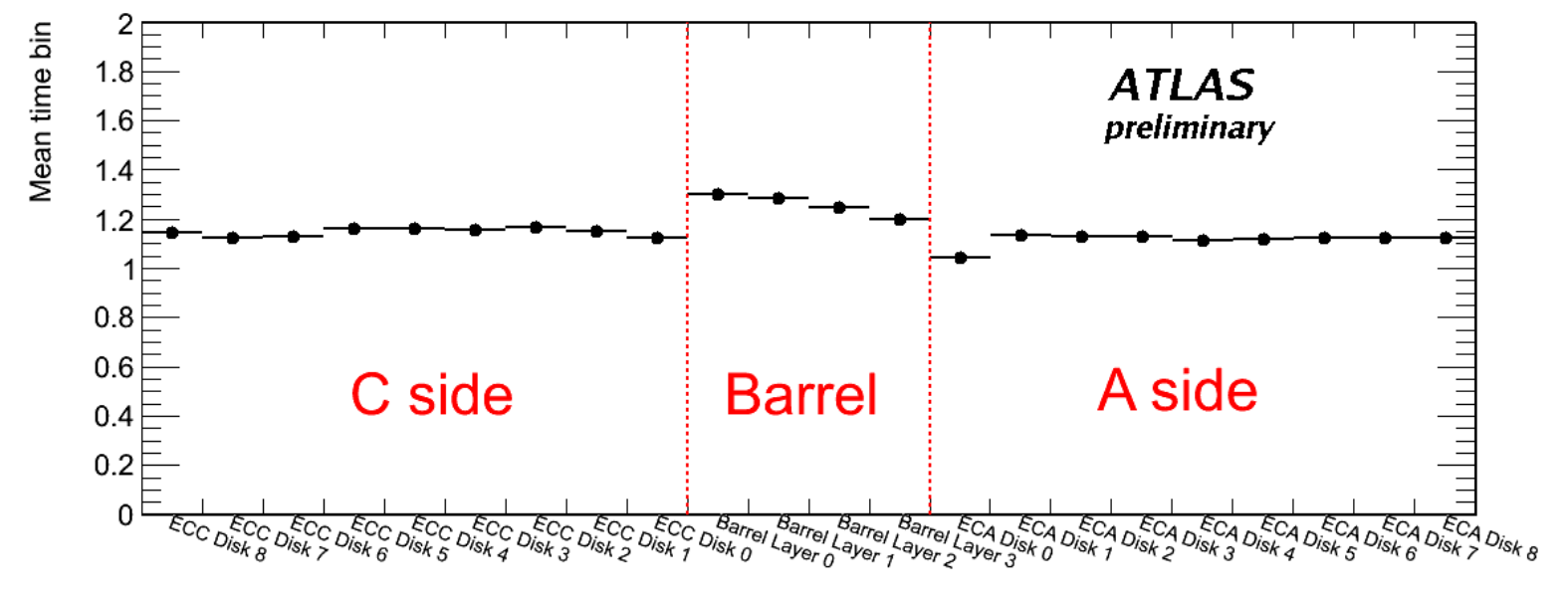




\section{Performance}

Number of Strips per Module Side (Occupancy)

- Data vs simulation

- Normalised by number of events

- All SCT modules

- Good agreement

- Discrepancy at low N (Due, in part, to there

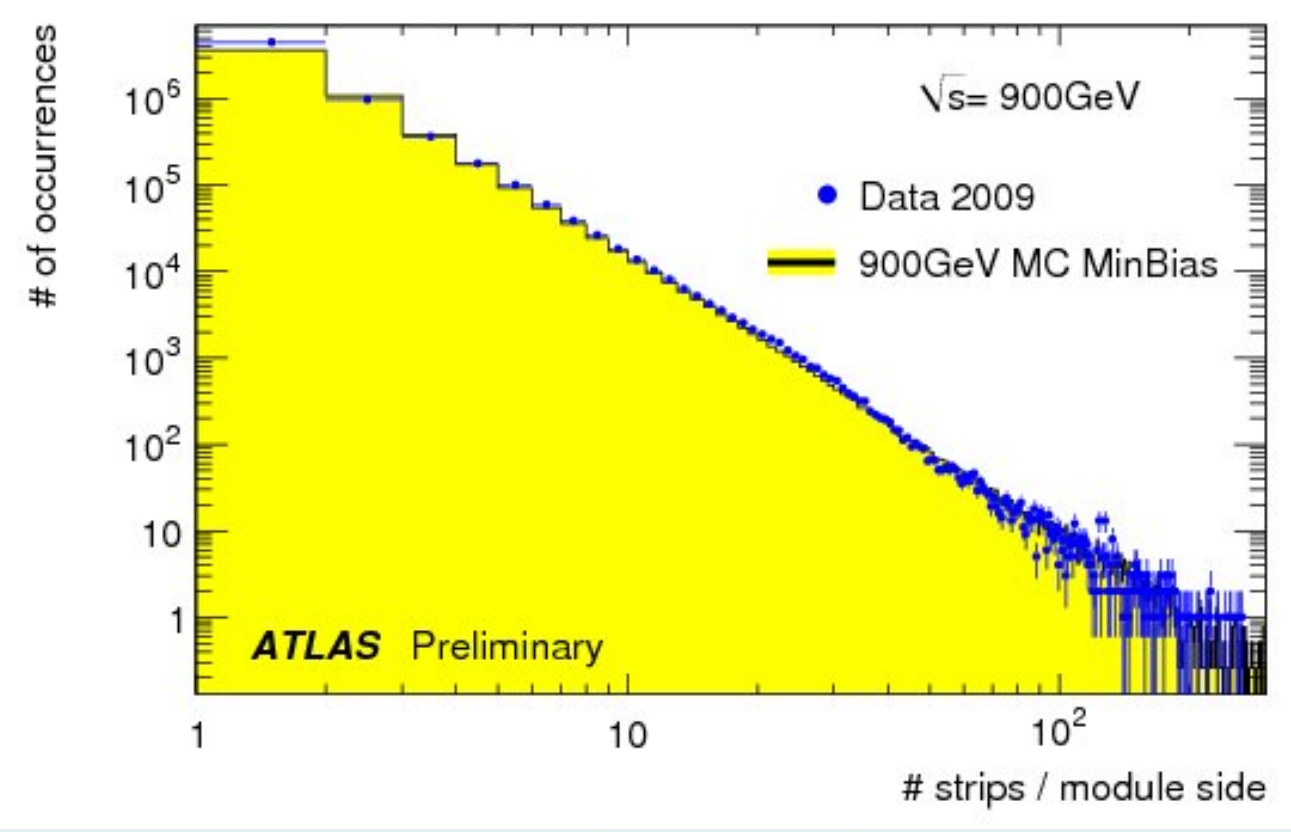
being more noise in data than Monte Carlo simulations) 


\section{Performance}

- Hit Efficiency

Intrinsic hit efficiency of the SCT measures the fraction of hits per possible hit For a well reconstructed track the number of hits (clusters) and the number of holes (where a hit is expected but not found) are counted Efficiency defined as:

$$
\varepsilon=\mathbf{N}_{\text {clusters }} /\left(\mathbf{N}_{\text {clusters }}+\mathbf{N}_{\text {holes }}\right)
$$

- Dead modules and chips taken in to account

- Tracks require $\geq 20$ TRT hits and $\geq 6$ SCT hits

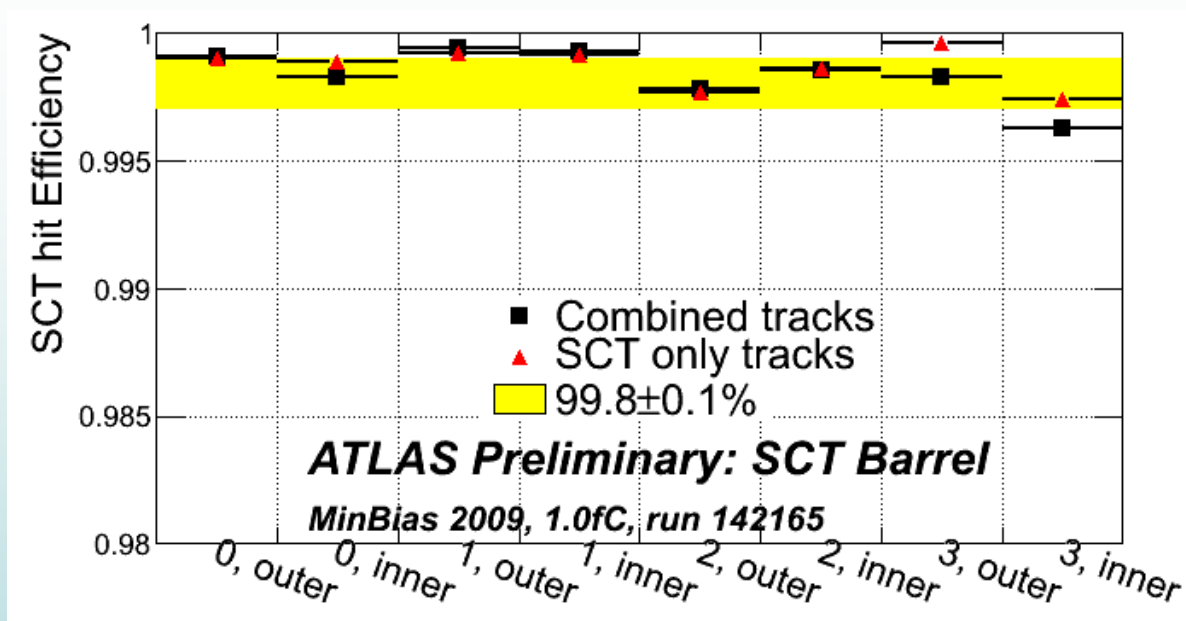




\section{Performance}

- Hit Efficiency for Endcaps A and C

- Dead modules and chips taken in to account

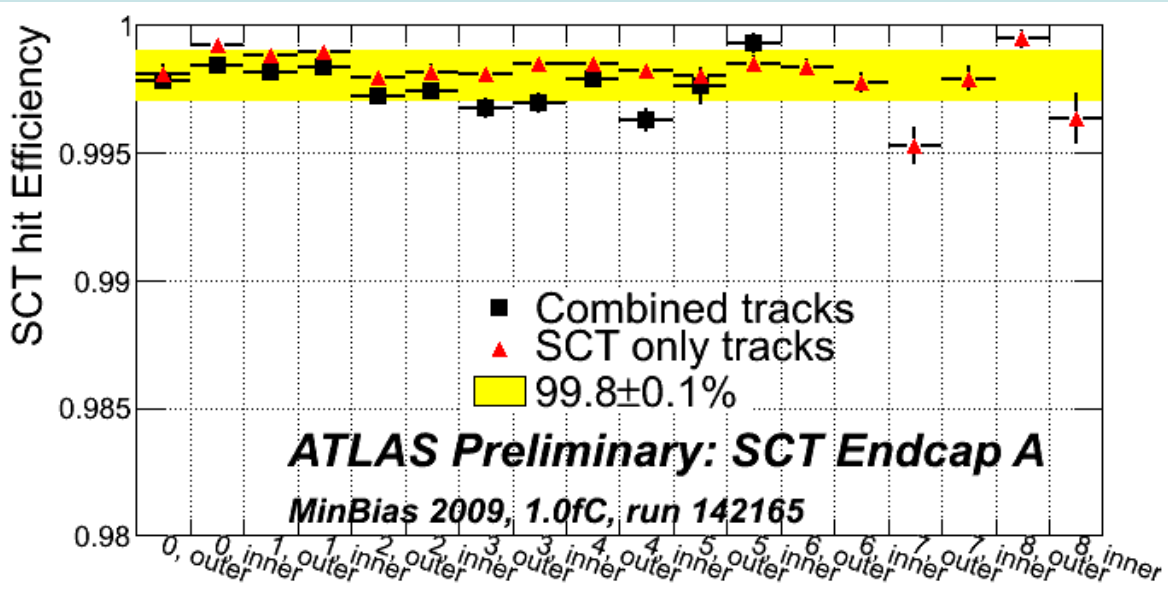

- Tracks require $\geq 20$ TRT hits and $\geq 6 \mathrm{SCT}$ hits

$\boldsymbol{\varepsilon}=\mathbf{N}_{\text {clusters }} /\left(\mathbf{N}_{\text {clusters }}+\mathbf{N}_{\text {holes }}\right)$

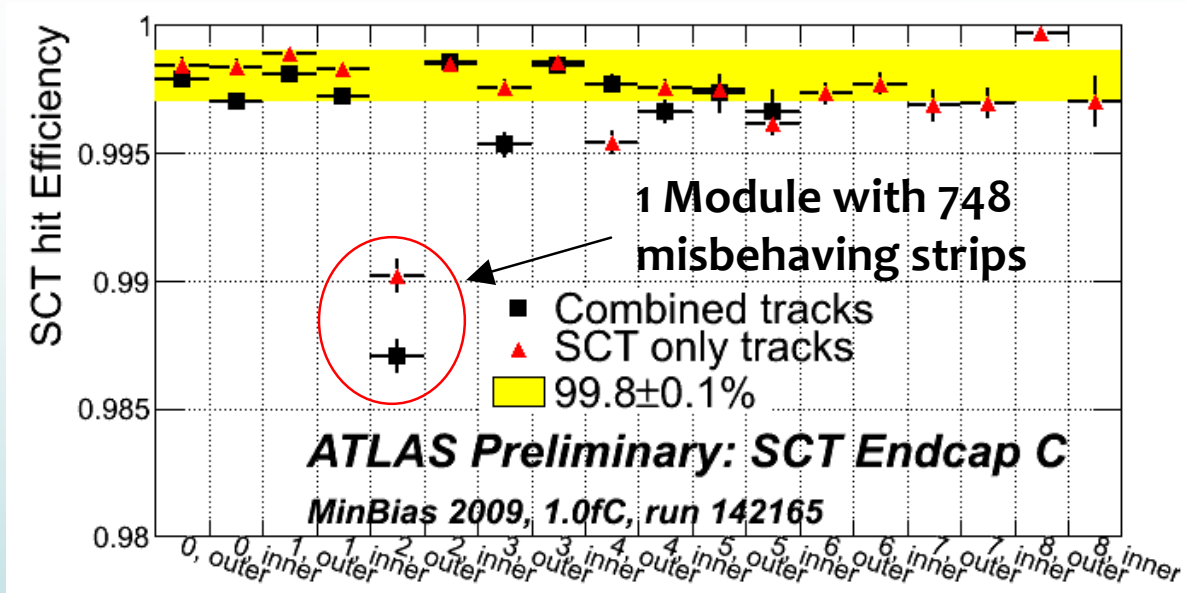




\section{Performance}

\section{Alignment}

- Residual = measured hit position - expected hit position (from the track extrapolation)

- As cosmics are vertical they are particularly useful to align the barrel

- With collision data the alignment of the endcaps are seen to improve (in particular SCT endcap C)

\section{Unbiased residual distribution for barrel}

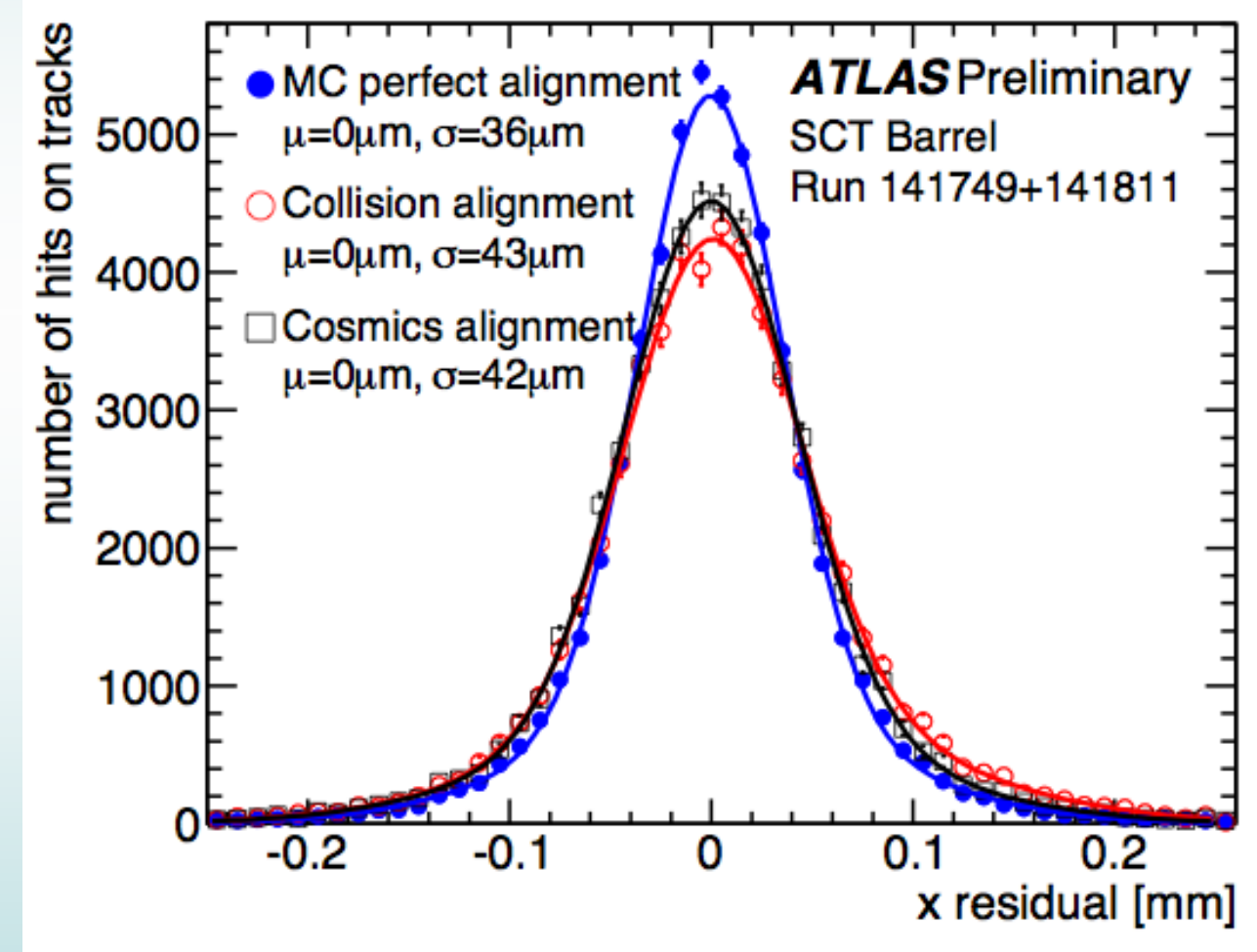




\section{Performance}

\section{Alignment}

- Residual = measured hit position - expected hit position (from the track extrapolation)

- As cosmics are vertical they are particularly useful to align the barrel

- With collision data the alignment of the endcaps are seen to improve (in particular SCT endcap C)

\section{Unbiased residual distribution for endcaps}

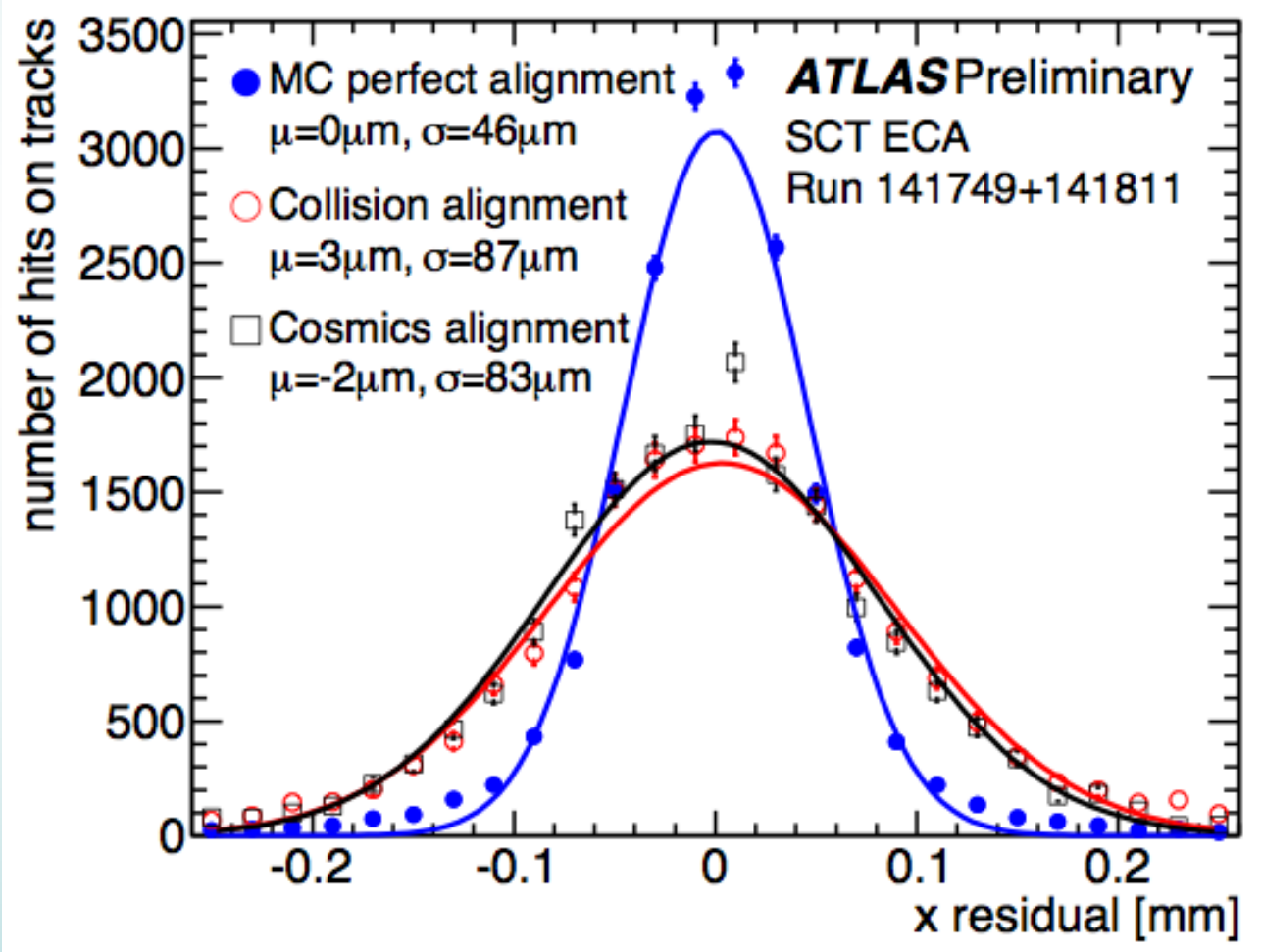




\section{Performance}

\section{Alignment}

- Residual = measured hit position - expected hit position (from the track extrapolation)

- As cosmics are vertical they are particularly useful to align the barrel

- With collision data the alignment of the endcaps are seen to improve (in particular SCT endcap C)

\section{Unbiased residual distribution for endcaps}

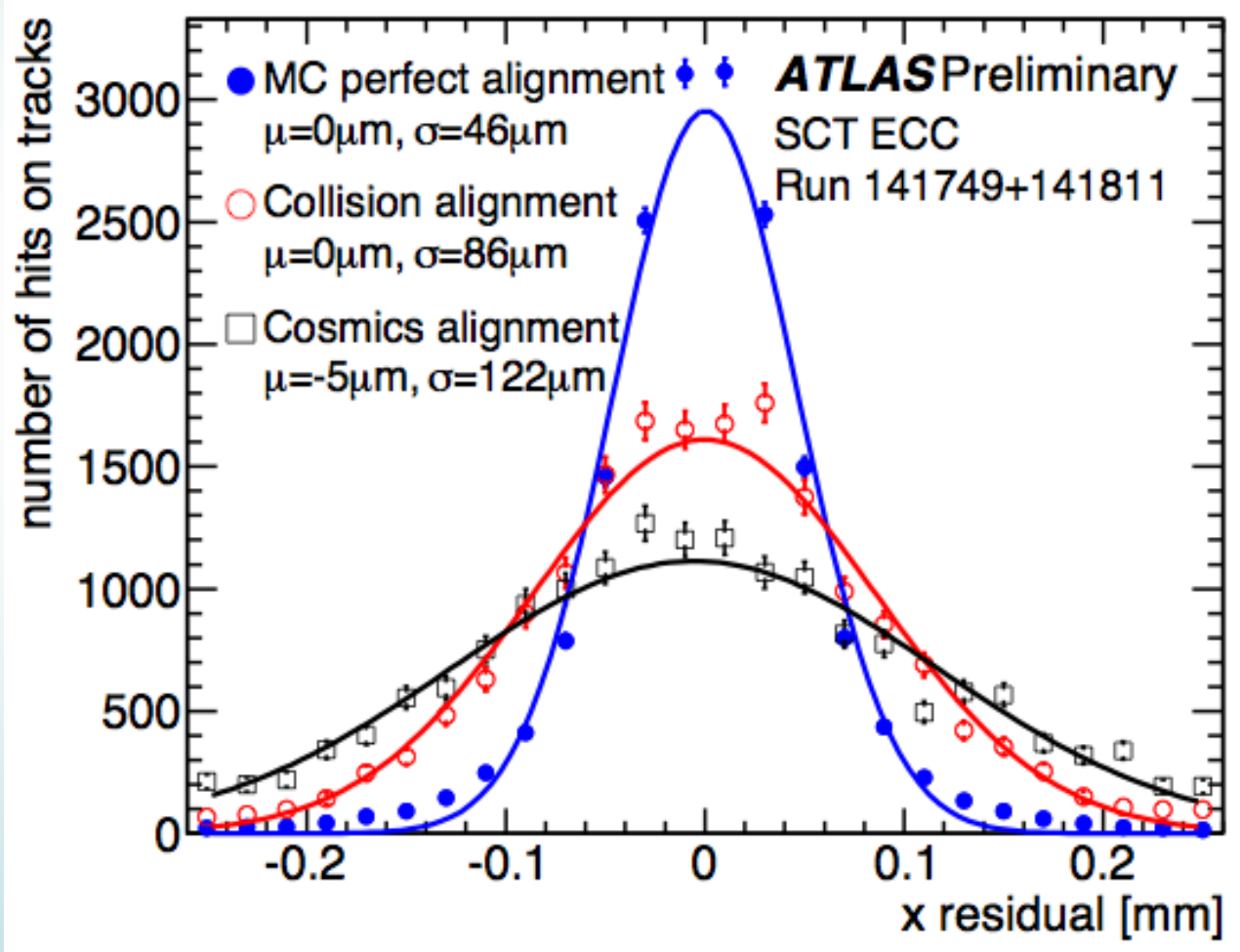




\section{Performance}

Alignment

- ID alignment further improved using $900 \mathrm{GeV}$ and then $7 \mathrm{TeV}$ collision data

- Alignment very close to ideal alignment (MC)

SCT Barrel:

Cosmics $(42 \mu \mathrm{m}), \operatorname{Cosmic} M C(36 \mu \mathrm{m})$ Collision $\operatorname{Data}(42 \mu \mathrm{m}), \mathrm{MC}(34 \mu \mathrm{m})$

\section{SCT Endcap:}

EndcapA: Cosmics $(83 \mu \mathrm{m}), \mathrm{MC}(46 \mu \mathrm{m})$

EndcapC: Cosmics $(122 \mu \mathrm{m}), \mathrm{MC}(46 \mu \mathrm{m})$

Collsion Data $(44 \mu \mathrm{m}), \mathrm{MC}(38 \mu \mathrm{m})$
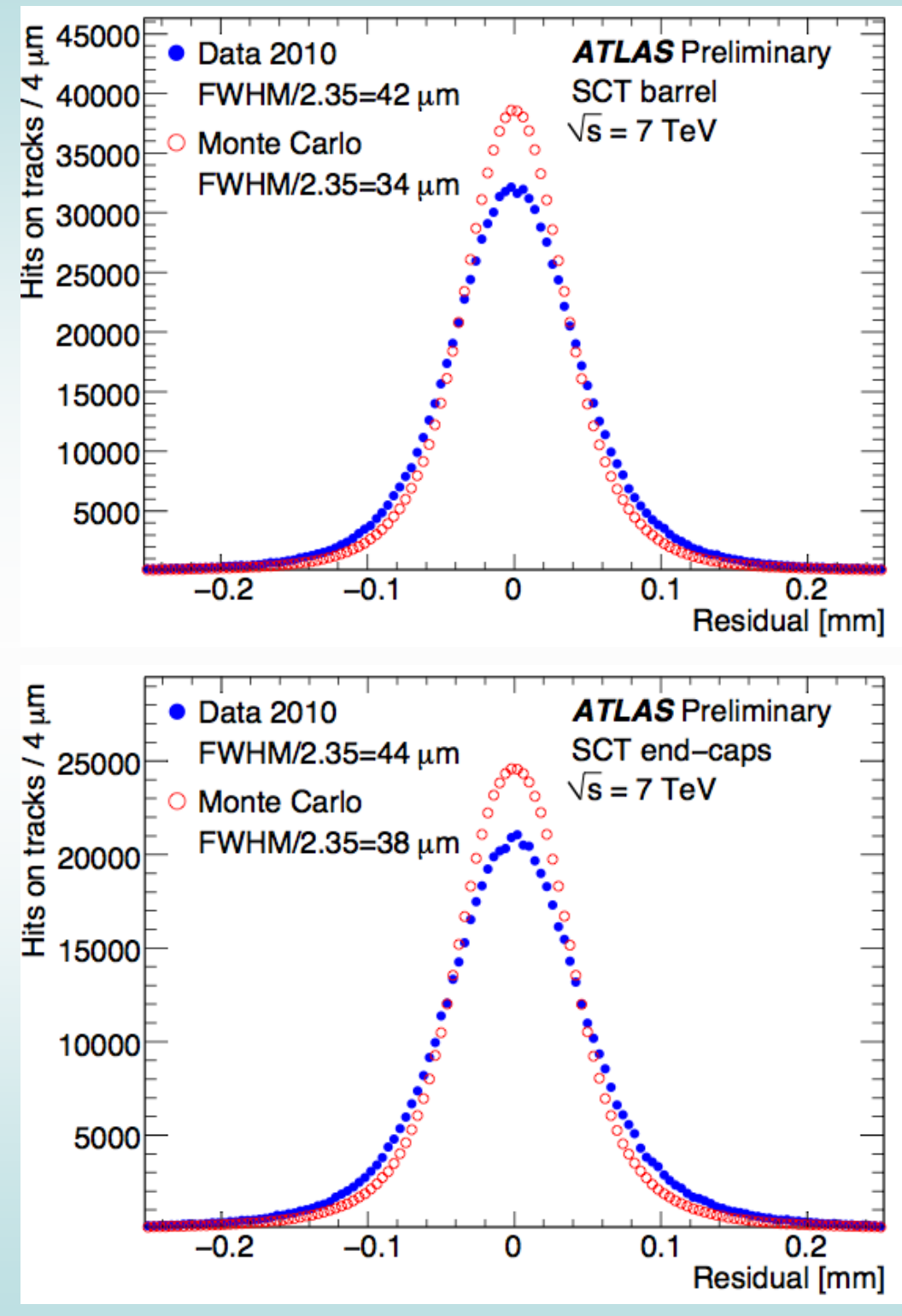


\section{Performance}

- Good alignment allows for accurate tracking and invariant mass reconstruction

- Plot shows the invariant mass distribution of 2 track (opposite charge) vertices

- Accurate reconstruction of $\mathrm{K}$ short invariant

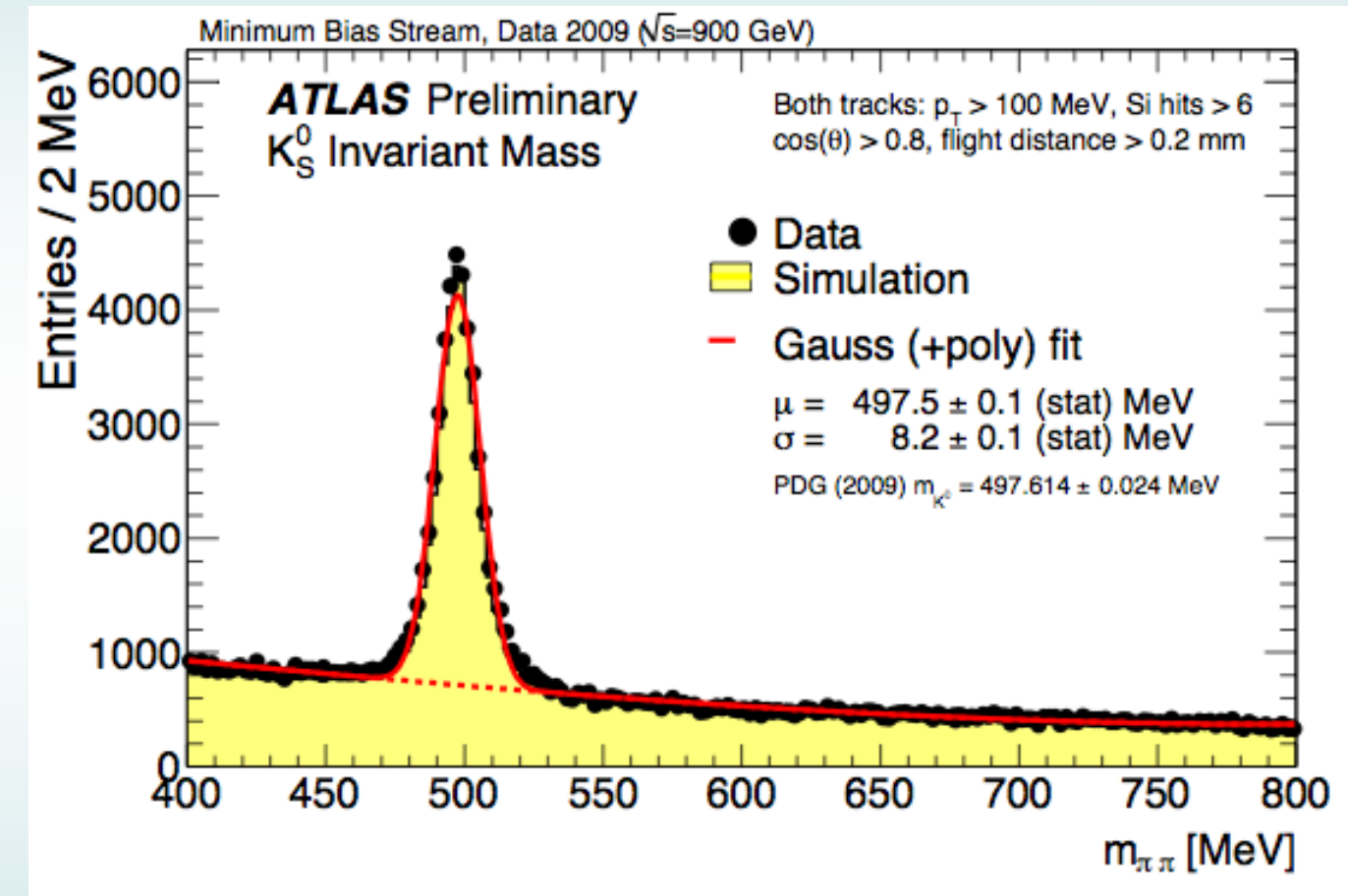
mass 


\section{Performance}

Lorentz Angle

- As charge carriers drift through the silicon wafer to the readout strips they are deflected by the magnetic field

- The Lorentz angle is the angular deflection relative to the electric field direction

- Depends on magnetic field strength, bias voltage across the wafer and temperature

- Model: $\theta_{\mathrm{L}}=-3.69^{\circ} \pm 0.26^{\circ}$

- Error on model from uncertainties in charge-carrier mobility

- From 2008 Cosmic data:

$\theta_{L}=-3.93^{\circ} \pm 0.03^{\circ} \pm 0.10^{\circ}$

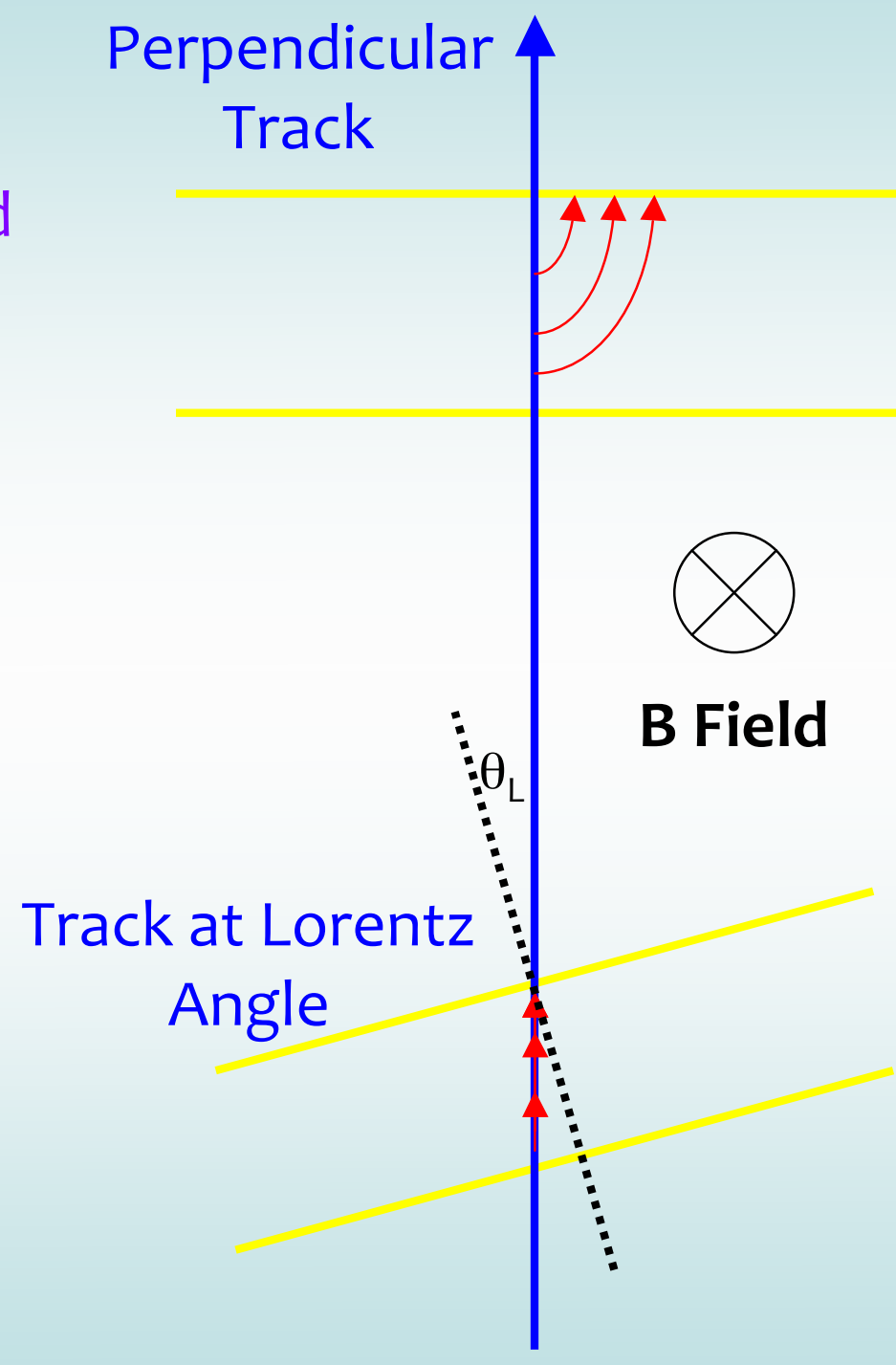

- Dominant systematic error from fit range

- Compatible with model 


\section{Performance}

\section{Lorentz Angle with collisions}

- Number of strips in a cluster vs incident angle of the track has minimum displacement from zero equal to the Lorentz angle

- Layers operate at different temps

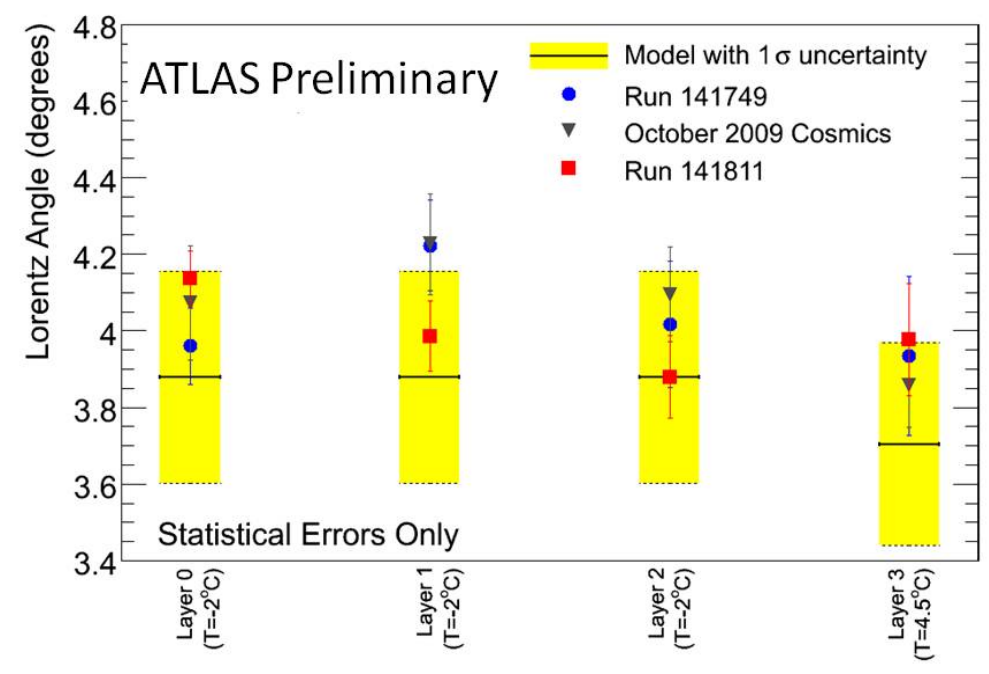

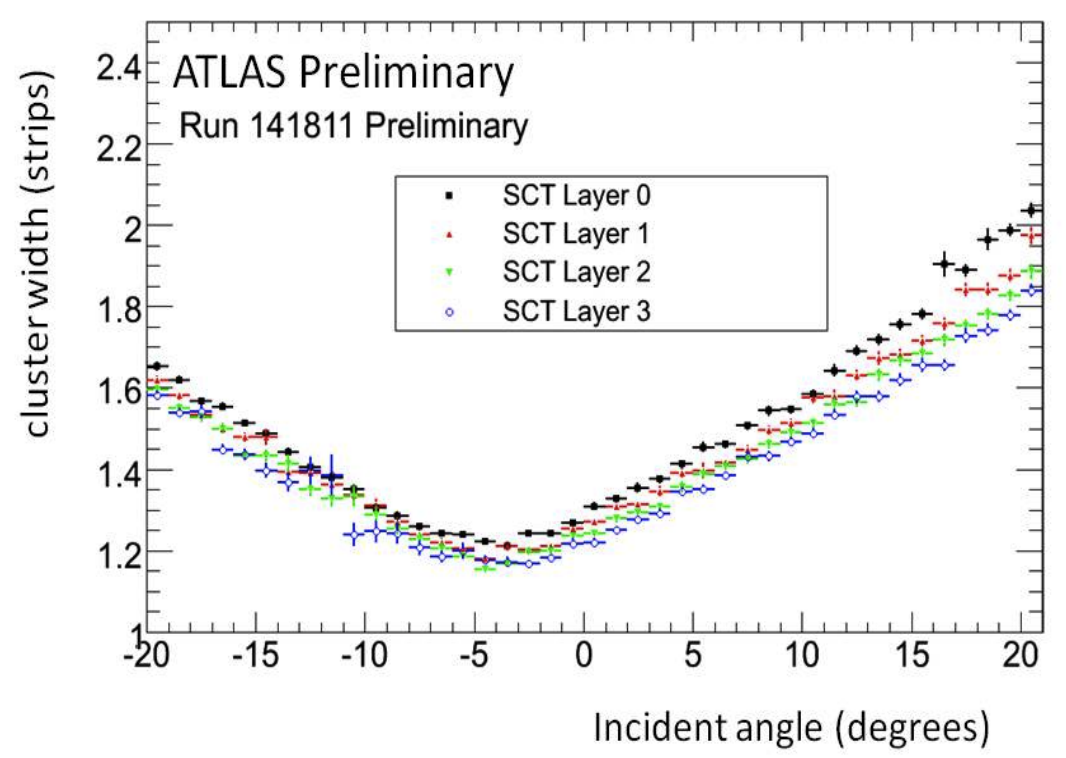

- Lorentz angle extracted from the cluster size vz angle distribution

- Shown for cosmic data, collision data and also the model prediction in yellow

- Measured value is higher than prediction by $\sim 1 \sigma$ of error on model prediction 


\section{Conclusions}

- The SCT has performed well during commissioning, cosmic data taking and collisions:

- 99.3\% of all SCT modules operational

- Noise occupancy and hit efficiency exceed design specifications

- Alignment very close to nominal, allowing for accurate track and invariant mass reconstruction

- Resolutions consistent with expectations

Exciting times ahead with greater luminosity, beams at higher intensity and ultimately higher energy collisions 


\section{Bonus Material: The Barrel Modules}

1. 4 Sensors

285 microns thick $p-n$

(Hamamatsu)

Strip length $12 \mathrm{~cm}$

Pitch $80 \mu \mathrm{m}$

$\mathrm{V} \max =500 \mathrm{~V}$

7. Overlaps

Overlap in $\mathrm{r} \phi$ and $\mathrm{Z}$ to

adjacent modules

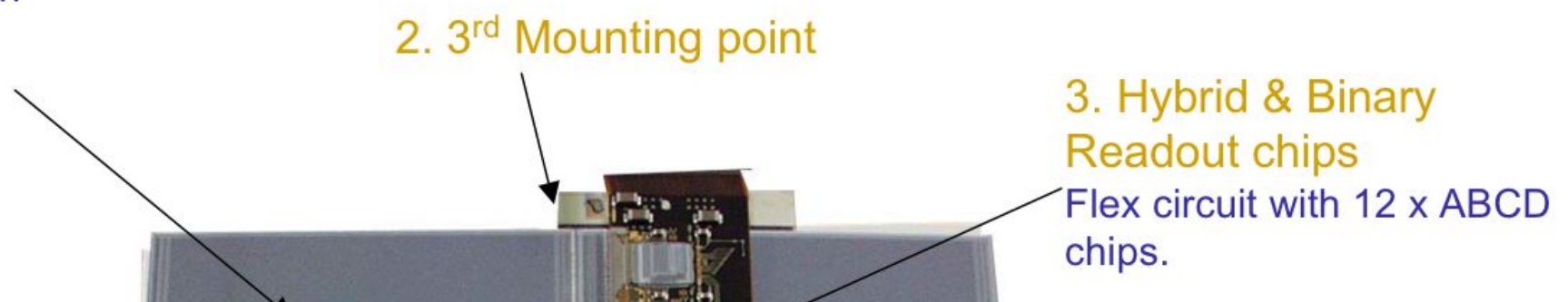

8. Stereo angle

Upper or lower detector pairs

rotated by $40 \mathrm{mRad}$

6. Connector

Power \& Data

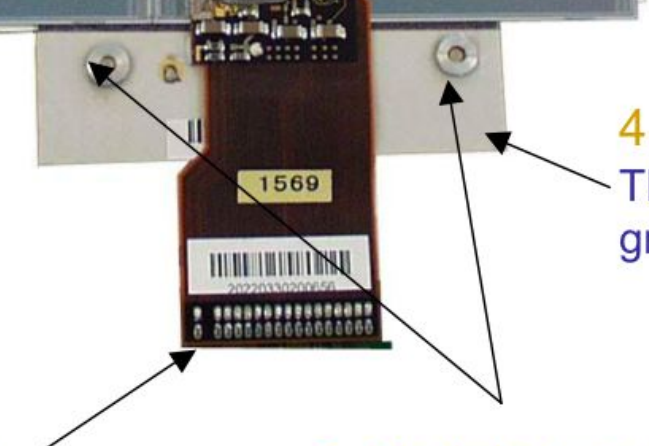

4. Be Facing \& Central TPG TPG (thermal pyrolythic graphite) plate for sensor cooling 


\section{Bonus Material: The Endcap Modules}

2. Hybrid \& Readout chips

1. 4 Sensors 280 microns thick (Hamamatsu, CIS) Strip length $12 \mathrm{~cm}$ Pitch 70-90um $\mathrm{V} \max =500 \mathrm{~V}$

8. Mounting point cooling interface TPG spine for sensor cooling

7. Stereo angle Upper or lower detector pairs rotated by $40 \mathrm{mrad}$

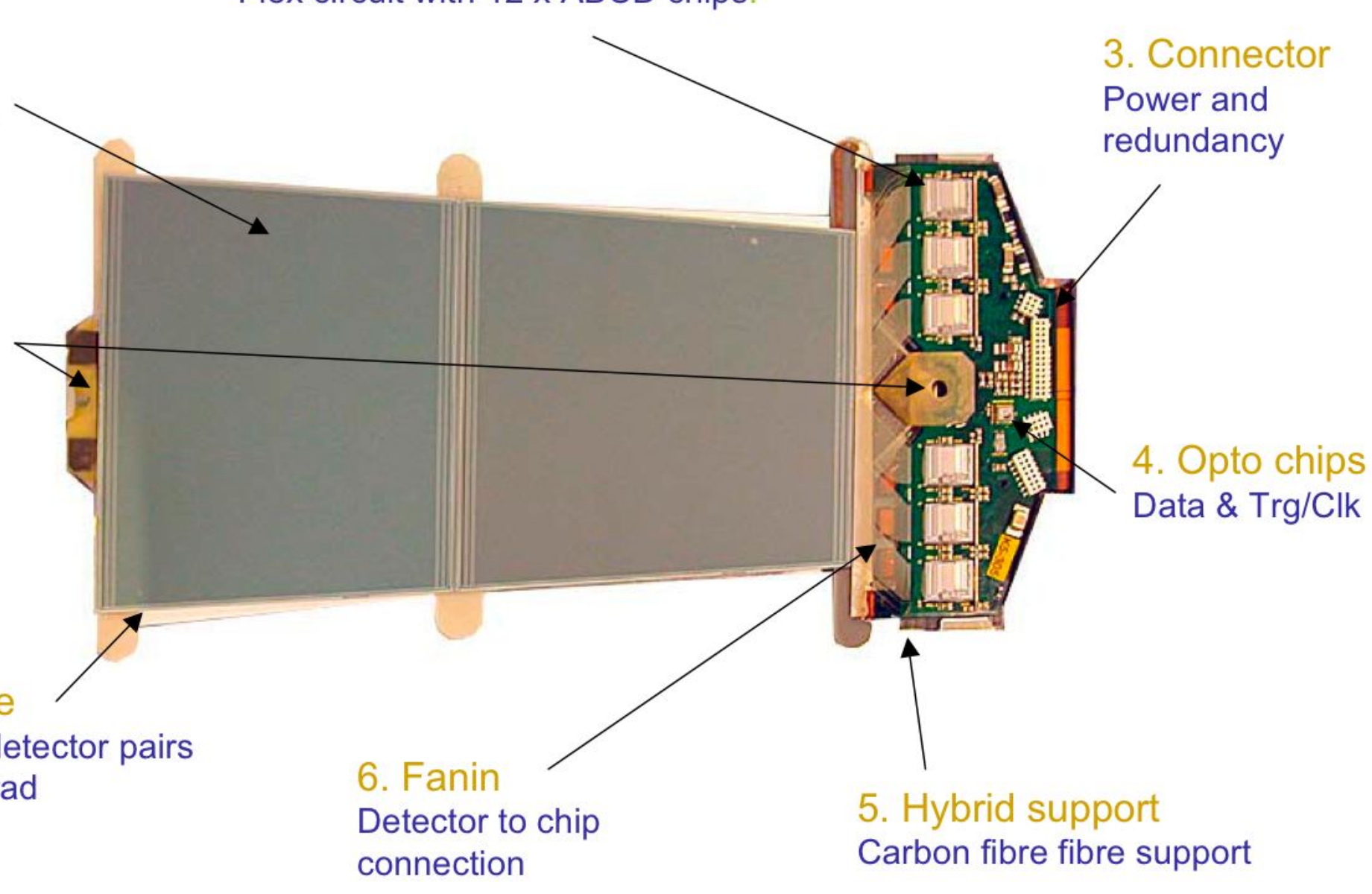

